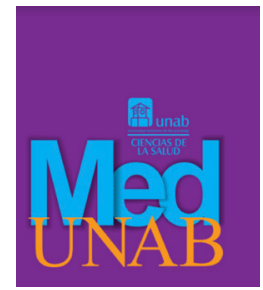

REVISTA DE LA FACULTAD

DE CIENCIAS DE LA SALUD

\title{
Tamización de síntomas de depresión, ansiedad prenatal y factores de riesgo psicosocial asociados en usuarias del servicio de control prenatal. Reflexiones en torno a la construcción del protocolo.
}

Screening for symptoms of depression, prenatal anxiety and psychosocial risk factors among users of prenatal control services. Reflections on creation of the protocol.

Triagem de sintomas de depressão, ansiedade pré-natal e fatores de risco psicossociais associados, em mulheres usuárias do serviço de controle pré-natal. Reflexões em relação à elaboração do protocolo.

Mónica Mojica-Perilla, Psi., MSc., PhD. ${ }^{1}$ (D), Yamilena Parra-Villa, Psi., MSc. ${ }^{2}$ (D), Sonia Esperanza Osma-Zambrano, MD., Esp., MSc. ${ }^{3}$ (D)

1. Psicóloga, Magíster en Educación, Doctora en Salud Pública. Universidad Autónoma de Bucaramanga. Bucaramanga, Santander, Colombia.

2. Psicóloga, Magíster en Psicología. Universidad Autónoma de Bucaramanga. Bucaramanga, Santander, Colombia.

3. Médica y Cirujana, Especialista en Ginecología y Obstetricia, Magíster en Epidemiología. Universidad Autónoma de Bucaramanga, Universidad Industrial de Santander. Bucaramanga, Santander, Colombia.

Correspondencia. Mónica Mojica-Perilla, Programa de Psicología, Universidad Autónoma de Bucaramanga. Calle 157 \# 19-55, Floridablanca, Santander, Colombia. Correo electrónico mmojica@unab.edu.co

Cómo citar. Mojica-Perilla M, Parra-Villa Y, Osma-Zambrano SE. Tamización de síntomas de depresión, ansiedad prenatal y factores de riesgo psicosocial asociados en usuarias del servicio de control prenatal. Reflexiones en torno a la construcción del protocolo. MedUNAB. 2019;22(3):341-355. doi: $10.29375 / 01237047.2867$

\section{INFORMACIÓN DEL ARTÍCULO.}

Artículo recibido: 24 de enero de 2018

Artículo aceptado: 31 de agosto de 2019

DOI: https://doi.org/10.29375/01237047.2867 


\section{RESUMEN}

Introducción. En los últimos años, los problemas de salud mental durante el período prenatal se han convertido en un tema prioritario para el campo de la salud pública. A los efectos adversos que un estado de salud mental alterado representa para el bienestar de la madre y su descendencia se suma una debilidad de los sistemas de salud para dar respuesta a esta situación. En Colombia son aún inciertas las cifras de prevalencia de cualquier problema de salud mental durante la gestación, lo que dificulta la posibilidad de ofrecer una atención que considere las particularidades del contexto. El objetivo es diseñar un protocolo de tamización de depresión y ansiedad prenatal y factores de riesgo psicosocial asociados dirigido a usuarias del servicio de control prenatal de una institución hospitalaria del oriente colombiano.

Metodología. La construcción del protocolo corresponde a un método formal de acuerdo a la clasificación realizada por el Ministerio de Protección Social de Colombia para las Guías de Práctica Clínica, incluye la revisión, síntesis y análisis de literatura sobre la ansiedad y la depresión prenatal (junto con los factores de riesgo psicosocial asociados) así como sobre las estrategias de detección y atención de las mismas. Esta revisión sirvió como base para la elaboración de una versión preliminar del protocolo que fue sometida a una revisión externa para verificar su validez, claridad y aplicabilidad, antes de proceder con el diseño de la versión final.

Resultados. En concordancia con lo reportado en la literatura, el protocolo incluye la aplicación de instrumentos para la identificación de síntomas de ansiedad, depresión y factores de riesgo psicosocial asociados, tales como variables sociodemográficas (edad, nivel educativo, ocupación e ingresos económicos), el soporte social percibido y la calidad de la relación de pareja, antecedentes psicológicos y psiquiátricos personales y familiares, factores relacionados con el embarazo como complicaciones o experiencias negativas previas, características de personalidad y vivencia de eventos adversos y estresantes de la vida.

Conclusiones. El protocolo da respuesta al vacío de identificación y atención a los problemas de salud mental de las gestantes.

Palabras clave:

Embarazo; Depresión; Ansiedad; Tamización; Impacto psicosocial.

\section{ABSTRACT}

Introduction. In recent years, mental health problems during the prenatal period have become a priority in the public health field. The weakness of the healthcare systems' response to this situation adds to the adverse effects that an altered state of mental health can pose for the well-being of the mother and her child. In Colombia, the figures of prevalence of any mental health problem during pregnancy are still unknown, which hinders the possibility of providing care that considers the specific nature of the context. The objective is to design a protocol to screen for depression, prenatal anxiety and related psychosocial risk factors among users of the prenatal control service of a hospital in east Colombia.

Methodology. The creation of the protocol consists of a formal method in accordance with the classification carried out by the Colombian Ministry of Social Protection for the Clinical Practice Guidelines. It includes the review, summary and analysis of literature on prenatal depression and anxiety (together with the related psychosocial risk factors), as well as the detection and response strategies for them. This review served as a basis to prepare a preliminary version of the protocol, which was subject to an external review to verify its validity, clarity and suitability, before proceeding with the design of the final version.

Results. In accordance with the literature, the protocol includes the application of instruments to identify symptoms of anxiety and depression, as well as related psychosocial risk factors, such as sociodemographic variables (age, level of 
education, occupation and financial income); the perceived social support and quality of the couple relationship; personal and family psychological and psychiatric history; factors related to pregnancy, such as previous complications or negative experiences; personality characteristics; and experience of adverse and stressful life events.

Conclusions. The protocol responds to the gap in the identification of and response to mental health problems among pregnant women.

Keywords:

Pregnancy, Depression; Anxiety; Screening; Psychosocial impact.

\section{RESUMO}

Introdução. Nos últimos anos, problemas de saúde mental durante o pré-natal vêm se tornando uma questão prioritária no campo da saúde pública. Somado aos efeitos adversos que um estado alterado de saúde mental representa para o bem-estar da mãe e de seus filhos, encontra-se uma fraqueza dos sistemas de saúde para responder a esta situação. Na Colômbia, as taxas de prevalência de qualquer problema de saúde mental durante a gravidez ainda são incertas, o que dificulta oferecer cuidados que considerem as particularidades do contexto. O objetivo é elaborar um protocolo de triagem para depressão e ansiedade pré-natal e fatores de risco psicossociais associados, direcionado a usuárias do serviço de controle pré-natal de um hospital no leste da Colômbia.

Metodologia. A elaboração do protocolo corresponde a um método formal. De acordo com a classificação feita pelo Ministério da Proteção Social da Colômbia para as Diretrizes de Prática Clínica, inclui a revisão, síntese e análise da literatura sobre ansiedade e depressão pré-natal (juntamente com os fatores de risco psicossociais associados), bem como sobre suas estratégias de detecção e atenção. Esta revisão serviu de base para a elaboração de uma versão preliminar do protocolo que foi submetida a uma revisão externa para verificar sua validade, clareza e aplicabilidade, antes de prosseguir com a elaboração da versão final.

Resultados. De acordo com o relatado na literatura, o protocolo inclui a aplicação de instrumentos para identificação de sintomas de ansiedade, depressão e fatores de risco psicossociais associados, como variáveis sociodemográficas (idade, escolaridade, ocupação e renda econômica), a identificação de apoio social e a qualidade do relacionamento do casal, antecedentes psicológicos e psiquiátricos pessoais e familiares, fatores relacionados à gravidez, como complicações ou experiências negativas anteriores, características da personalidade e vivência de eventos adversos e estressantes da vida.

Conclusões. O protocolo responde à lacuna de identificação e atenção aos problemas de saúde mental de mulheres grávidas.

Palavras-chave:

Gravidez; Depressão; Ansiedade; Triagem; Impacto psicossocial. 


\section{Introducción}

Los riesgos inherentes al sexo femenino en el campo de la salud mental se tornan evidentes durante el período prenatal y posnatal; tiempo en el que los cambios biológicos y psicosociales a los que se ve sometida la futura madre pueden traspasar las barreras de adaptación normal a esta etapa y desembocar en alteraciones mentales. Aunque el embarazo es usualmente considerado como un periodo de relativo bienestar mental, las alteraciones hormonales pueden incrementar la vulnerabilidad a los desórdenes mentales (1).

Existe evidencia considerable que parte de muchos estudios prospectivos de que, si la madre está deprimida, ansiosa o estresada durante el embarazo, es más probable que su hijo experimente un neurodesarrollo con resultados adversos, en comparación a los casos de otras madres sin tales condiciones (2). Estos resultados incluyen, entre otros, un mayor riesgo de problemas emocionales, conductuales y cognitivos del niño, bajo peso al nacer, parto prematuro, retraso en el crecimiento y restricción en el crecimiento fetal (3-11).

El estrés, la depresión y la ansiedad afectan entre un $15 \%$ y $20 \%$ de las mujeres gestantes en el mundo. Sin embargo, poco menos del $20 \%$ de los proveedores de cuidado prenatal evalúan e intervienen los problemas de salud mental, y menos del $20 \%$ busca atención para trastornos mentales. Para aquellas que buscan tratamiento, la falta de integración al sistema de salud y las barreras existentes frecuentemente dificultan el acceso a un tratamiento. Sin intervención, una pobre salud mental prenatal puede persistir por años e impactar el bienestar futuro de la madre, del niño y del resto de la familia (12).

A pesar de las recomendaciones de la Organización Mundial de la Salud (OMS) y la creciente evidencia de alta prevalencia de problemas de salud mental materna, así como el impacto adverso de estos en la madre y su bebé, la agenda de salud mental materna no se ha incorporado en el sistema de atención primaria en la mayoría de los países de bajos y medianos ingresos (grupo en el que se encuentra incluida Colombia). Las barreras para la provisión de atención primaria de salud mental materna en estos países incluyen la falta de recursos humanos y financieros $\mathrm{y}$, con bastante frecuencia, la doble carga de enfermedades transmisibles y no transmisibles que relegan la salud mental a los márgenes de la atención médica. En consecuencia, hay una insuficiencia en el tratamiento para los trastornos mentales en estos países que va desde el $76 \%$ hasta el
$85 \%$ de los usuarios con problemas de salud mental que no reciben intervención(13).

Dado que, para el caso colombiano, la atención en salud mental prenatal es aún un tema poco explorado, se torna relevante ampliar el espectro de atención en este campo. Se requiere que dicha atención vaya más allá de los controles prenatales tradicionales e incluya una evaluación psicosocial inicial $\mathrm{y}$, posteriormente, una valoración psicológica o psiquiátrica para identificar e intervenir los factores de riesgo asociados al desarrollo y mantenimiento de los diferentes problemas de salud mental ya referidos. Lo anterior requiere el diseño de protocolos de atención que sean sensibles a la realidad nacional y regional, y que permitan la identificación temprana de factores de riesgo psicosocial y sintomatología asociados con la depresión y la ansiedad. Esto se convierte en una forma estratégica de prevención que tendrá un impacto positivo tanto en la salud de la madre y de su descendencia como en el clima familiar. Una vez identificadas las mujeres en riesgo, el sistema de salud debería realizarles un seguimiento adecuado que contemple la mitigación del impacto de los riesgos identificados, así como la remisión a profesionales especializados de la salud mental (psicólogos clínicos o psiquiatras, según la severidad del caso).

Es innegable la necesidad de atender las posibles dificultades de salud mental que pueden presentar las mujeres en estado de gestación, por lo que se hace necesario diseñar estrategias que respondan a las particularidades de cada región. Para el caso específico de Bucaramanga, se realizó un estudio con 244 gestantes con un promedio de edad de 24.8 años. Los resultados indican que la prevalencia de depresión fue de $24.6 \%$, IC $95 \%$ (19.1-30.0) y la de ansiedad $25.8 \%$, IC $95 \%$ (20.3-31.3). La depresión está asociada con antecedente familiar de depresión en primer o segundo grado, razón de prevalencia: 2.0, IC 95\% (1.1-3.7); presencia de ansiedad, razón de prevalencia: 22.5, IC 95\% (9.453.7); y consumo de alcohol, razón de prevalencia: 2.9, IC 95\% (1.1-8.2). Como factor protector se encontró tener dos fuentes de ingresos (pareja y familia), razón de prevalencia: 0.6, IC 95\% (0.4-0.8). Adicionalmente, la ansiedad se asoció con presencia de depresión, razón de prevalencia: 13.3, IC 95\% (6.3-28.1); presencia de violencia psicológica, razón de prevalencia: 2.3 , IC $95 \%$ (1.1-4.8) y no tener confianza en la pareja, razón de prevalencia: 3.4 , IC 95\% (1.5-8.2) (14). Con base en estos resultados, así como en la inexistencia de protocolos locales que guíen las acciones en el cuidado de la salud mental de las gestantes, surgió el proyecto que tuvo como objetivo el diseño de un protocolo de tamización de síntomas de depresión y ansiedad prenatal 
y factores de riesgo psicosocial asociados, dirigido a usuarias del servicio de control prenatal realizado en el Hospital Local del Norte, el cual atiende a población que reside principalmente en el sector norte de la ciudad, que es una zona vulnerable y posible beneficiaria de acciones encaminadas a mejorar el estado de salud y la calidad de vida de sus habitantes gestantes.

De esta manera, el presente artículo muestra la experiencia que llevó a la generación del protocolo de tamización y las reflexiones surgidas durante el mismo; proceso dinámico que permite afianzar el trabajo investigativo en salud mental materna iniciado hace cuatro años.

\section{Metodología}

El protocolo se diseñó atendiendo al proceso de construcción de guías de práctica clínica propuesto por el Ministerio de Protección Social de Colombia (15). Se pueden encontrar tres tipos diferentes: guías diseñadas por consenso informal (método informal o juicio global subjetivo), guías diseñadas por consenso formal -que no tienen la sistematización de la Medicina Basada en la Evidencia (MBE) - y finalmente, guías basadas en la evidencia (aplicación de la MBE), cada una de estas con sus características particulares (Tabla 1). Tomando como base estas características, la construcción del protocolo de tamización corresponde principalmente a un método formal. Aunque la revisión y el análisis de la literatura no corresponde a una revisión sistemática, sí fue exhaustiva y fundamental, ya que sobre esta base se tomaron decisiones relacionadas con: elección y peso de los factores de riesgo a identificar, pruebas de tamización a emplear y estrategias de acción a seguir por parte del profesional de la salud. En este orden de ideas, el procedimiento general que se empleó durante el diseño del protocolo fue adaptado de los lineamientos propuestos por Gouda, Hasman, Strijbis \& Peek (16) y Swinglehurst (17), y contempló las siguientes etapas: a) conformación de un equipo de trabajo interdisciplinario, b) revisión de la literatura, que tuvo como objetivo sintetizar y analizar la información disponible sobre el área temática del protocolo, c) elaboración de una versión preliminar del protocolo, d) sometimiento de la versión preliminar a revisión por parte de los potenciales usuarios para verificar la validez, claridad y aplicabilidad, y, por último, e) elaboración de la versión final del protocolo.

La primera versión del protocolo fue socializada en una reunión a la que asistieron dos docentes ginecoobstetras de la Universidad Autónoma de Bucaramanga, quienes realizan actividades de docencia y servicio en el Hospital Local del Norte, además de seis residentes de la Especialización en Ginecología y Obstetricia de la misma universidad. En dicha reunión, el equipo investigador socializó el proceso de construcción del protocolo, entregando una copia de este y un formato para evaluar su validez de contenido. Después de diligenciar dicho formato los asistentes manifestaron

Tabla 1. Diferencias entre los tipos de Guías de práctica clínica.

\begin{tabular}{llll}
\hline Características & GPC método informal & GPC método formal & $\begin{array}{l}\text { GPC basada en la } \\
\text { evidencia }\end{array}$ \\
\hline Sustento principal & $\begin{array}{l}\text { Experiencia de los } \\
\text { participantes }\end{array}$ & $\begin{array}{l}\text { Experiencia de los } \\
\text { participantes }\end{array}$ & Método científico \\
\hline $\begin{array}{l}\text { Investigación } \\
\text { bibliográfica }\end{array}$ & No necesaria & $\begin{array}{l}\text { Investigación } \\
\text { bibliográfica no } \\
\text { sistematizada }\end{array}$ & $\begin{array}{l}\text { Consulta de bases } \\
\text { médicas informatizadas }\end{array}$ \\
\hline $\begin{array}{l}\text { Análisis crítico de la } \\
\text { literatura }\end{array}$ & No necesario & No sistematizado & Fundamental \\
\hline $\begin{array}{l}\text { Variabilidad en sus } \\
\text { recomendaciones }\end{array}$ & Muy amplia & Amplia & Mínima \\
\hline Actualización & No necesaria & Escasa & Por definición \\
\hline
\end{tabular}

Fuente: Ministerio de la Protección Social, Colciencias, Centro de Estudios e Investigación en Salud de la Fundación Santa Fe de Bogotá, Escuela de Salud Pública de la Universidad de Harvard. Guía Metodológica para el desarrollo de Guías de Atención Integral en el Sistema General de Seguridad Social en Salud Colombiano. Bogotá, Colombia 2010. 
su opinión sobre el protocolo y realizaron algunas sugerencias al mismo, que fueron tenidas en cuenta para la redacción de la versión final. En términos generales, los asistentes consideraron que el protocolo es relevante para su práctica clínica; que está redactado con claridad y que está organizado de manera lógica, coherente y secuencial. Igualmente, que la información suministrada es suficiente para desarrollar la tarea de tamización y, por lo tanto, permite el logro de su objetivo.

\section{Resultados}

\section{Detección de síntomas de depresión, ansiedad prenatal y factores de riesgo asociados por medio de evaluación psicosocial}

Los resultados de la revisión de la literatura se pueden agrupar en dos grandes apartados. En primer lugar, se encuentra la información relacionada con la identificación de las principales dificultades de salud mental reportadas para el período perinatal. A partir de estos resultados el equipo toma la decisión de incluir en el diseño del protocolo únicamente la identificación de síntomas de depresión y ansiedad, considerando que su detección en el período prenatal permite un seguimiento adecuado, mejorando así los resultados de cuidado de la salud mental para las mujeres en estado de gestación. Lo anterior requiere un enfoque que reconozca que el estrés propio del embarazo puede convertirse en depresión o ansiedad y que la gravedad de los síntomas puede aumentar durante el período perinatal (18). La identificación de síntomas también viene asociada a la delimitación de factores de riesgo psicosocial, cuya tipificación es esencial en las tareas de prevención. Dentro de dichos factores de riesgo, la literatura reporta como relevantes las siguientes variables: condición sociodemográfica (edad, nivel educativo, ocupación, ingresos económicos), soporte social percibido y calidad de la relación de pareja, antecedentes psicológicos y psiquiátricos (personales y familiares), factores relacionados con el embarazo como complicaciones o experiencias negativas previas, características de personalidad y experiencias de eventos adversos o estresantes de la vida (Tablas 2-4).

Algunos autores plantean que la administración de instrumentos de tamización para identificar mujeres en riesgo durante el embarazo debería ser una práctica universal. De esta forma se podría promover el bienestar

Tabla 2. Factores de riesgo para el desarrollo de trastornos de ansiedad durante el período perinatal.

\begin{tabular}{ll}
\hline Categoría & Factores de riesgo \\
\hline Factores económicos y socio demográficos & Edad materna: jóvenes \\
& Soltera - sin pareja \\
& Bajo nivel escolaridad de la madre \\
& Nivel socio económico bajo: desempleo, bajos ingresos, \\
& Adversidad financiera \\
& Madre primigesta \\
\hline Factores sociales & Problemas en la relación de pareja \\
& Pobre apoyo social \\
& Violencia doméstica - física \\
& Estresores de vida recientes \\
\hline Experiencia del embarazo y parto & Perdidas perinatales previas \\
& Embarazo no planeado \\
& No asistencia a curso prenatal \\
& Pobre salud durante el embarazo \\
\hline Factores psicológicos & Baja autoestima \\
& Ansiedad rasgo alta \\
& Historia de problemas psiquiátricos \\
& Actitudes negativas hacia el embarazo y él bebe \\
& Historia previa de trauma \\
\hline
\end{tabular}

Fuente: Leach LS, Poyser C, Fairweather-Schmidt K. Maternal perinatal anxiety: A review of prevalence and correlates. Clinical Psychologist 2017; 21(1): 4-19. doi: 10.1111/cp.12058 
Tabla 3. Factores de riesgo para el desarrollo de trastornos de depresión durante el período perinatal.

\begin{tabular}{|c|c|c|}
\hline Categoría & $\begin{array}{l}\text { Factores de riesgo (Lancaster CA et } \\
\text { al) }\end{array}$ & $\begin{array}{l}\text { Factores de riesgo (Pereira PK, } \\
\text { Lovisi GM) }\end{array}$ \\
\hline Factores Personales & Embarazo no deseado & $\begin{array}{l}\text { Actitud negativa frente a la gravidez } \\
\text { Embarazo no deseado } \\
\text { Percepción negativa de su estado de } \\
\text { salud } \\
\text { Estrategias de afrontamiento } \\
\text { Abortos anteriores }\end{array}$ \\
\hline Factores psicológicos & $\begin{array}{l}\text { Ansiedad } \\
\text { Historia personal de depresión }\end{array}$ & $\begin{array}{l}\text { Antecedentes psiquiátricos } \\
\text { Ansiedad } \\
\text { Estrés } \\
\text { Ideación suicida }\end{array}$ \\
\hline Situaciones estresantes & $\begin{array}{l}\text { Eventos negativos de la vida (crisis) } \\
\text { Molestias diarias }\end{array}$ & Eventos estresantes \\
\hline Soporte social & $\begin{array}{l}\text { Apoyo social de cualquier fuente } \\
\text { Apoyo social de la pareja }\end{array}$ & $\begin{array}{l}\text { Vivir sola } \\
\text { Ser soltera, divorciada o separada } \\
\text { Bajo soporte social } \\
\text { Familias desestructuradas } \\
\text { Poco soporte marital } \\
\text { Inestabilidad en las relaciones }\end{array}$ \\
\hline Violencia & Violencia domestica & $\begin{array}{l}\text { Historia de violencia doméstica } \\
\text { Conflictos conyugales } \\
\text { Violencia marital } \\
\text { Violencia física }\end{array}$ \\
\hline $\begin{array}{l}\text { Relaciones } \\
\text { interpersonales }\end{array}$ & $\begin{array}{l}\text { Mala calidad de las relaciones } \\
\text { interpersonales }\end{array}$ & $\begin{array}{l}\text { Conflictos familiares } \\
\text { Dificultades en las relaciones sociales }\end{array}$ \\
\hline Demográficos & $\begin{array}{l}\text { Estrato socioeconómico } \\
\text { Bajos ingresos } \\
\text { Desempleo } \\
\text { Baja escolaridad }\end{array}$ & $\begin{array}{l}\text { Baja calidad de vida } \\
\text { Dificultades financieras } \\
\text { Baja escolaridad } \\
\text { Baja renta familiar } \\
\text { Desempleo } \\
\text { Pobreza }\end{array}$ \\
\hline Uso de sustancias & $\begin{array}{l}\text { Uso de alcohol } \\
\text { Fumar }\end{array}$ & $\begin{array}{l}\text { Uso de alcohol } \\
\text { Fumar }\end{array}$ \\
\hline
\end{tabular}

Fuente: Pereira PK, Lovisi GM. Prevalência da depressão gestacional e fatores asociados. Rev Psiq Clín 2008; 35(4): 144-53. http://dx.doi.org/10.1590/S0101-60832008000400004 Lancaster CA, Gold KJ, Flynn HA, Yoo H, Marcus SM, Davis M. Risk factors for depressive symptoms during pregnancy: a systematic review. Am J Obstet Gynecol 2010; 202(1): 5-14. doi: 10.1016/j.ajog.2009.09.007.

a largo plazo, tanto del bebé como de la madre. En este sentido, el conocimiento de los factores de riesgo específicos puede ayudar a crear dicha herramienta de detección dirigida a las mujeres con mayor riesgo. Es así como la correcta identificación de estas mujeres permitiría que se beneficiaran de intervenciones preventivas, al igual que de apoyo e intervenciones terapéuticas, si el caso lo requiriese (19).

La depresión prenatal, la ansiedad y el estrés están severamente subdetectados $\mathrm{y}$, por lo tanto, su tratamiento es escaso. Adicionalmente, dos tercios de las mujeres con síntomas importantes o de significancia clínica no 
Tabla 4. Factores de riesgo para el desarrollo de trastornos depresión y ansiedad durante el período perinatal.

\begin{tabular}{ll}
\hline Categoría & Factores de riesgo para ansiedad y depresión \\
\hline Factor eventos estresantes & Eventos adversos en la vida y percepción de estrés \\
\hline Factores psicológicos y & Historia personal de trastornos mentales/Tratamiento psiquiátrico \\
psiquiátricos & Consumo de alcohol actual o pasado \\
& Consumo de cigarrillo actual o pasado \\
& Uso de sustancias psicoactivas durante el embarazo \\
& Abuso infantil \\
& Calidad o estilo de crianza durante la niñez \\
& Historia familiar de trastornos mentales \\
\hline Factores de soporte social y & Falta de pareja o soporte social \\
relaciones maritales & Conflictos sociales \\
& Problemas o insatisfacción con la relación de pareja \\
& Historia de abuso o violencia domestica \\
& Madre soltera o no convivir con la pareja \\
\hline Factores sociodemográficos y & Temprana edad o ser muy joven \\
económicos & Edad avanzada \\
& Bajo nivel educativo \\
& Desempleo \\
& Ser ama de casa \\
& Dificultades en el lugar de trabajo \\
& Desempleo de la pareja \\
& Bajos ingresos y dificultades financieras \\
\hline Factores de personalidad & Embarazo no planeado \\
\hline Factores obstétricos y & Multiparidad \\
& Presentes o pasadas complicaciones durante el embarazo o pérdidas \\
& Historia de episiotomías, cesáreas, experiencias negativas en el \\
& nacimiento, tratamientos para la infertilidad, temor al parto. \\
\hline & Autoeficacia y autoestima negativas \\
\hline
\end{tabular}

Fuente: Biaggi A, Conroy S, Pawlby S, Pariante CM. Identifying the women at risk of antenatal anxiety and depression: A systematic review. Journal of Affective Disorders 2016; 19: 62-77. doi:10.1016/j.jad.2015.11.014.

son identificadas por la mayoría de los trabajadores de la salud que se desempeñan en al área obstétrica $(20,21)$. La detección universal se está considerando cada vez la mejor estrategia en muchos entornos para la identificación temprana y la intervención oportuna, por lo que es esencial que los profesionales de la salud cuenten con entrenamiento en una evaluación psicosocial más amplia que maximice la utilidad y reduzca al mínimo los daños potenciales. La evaluación psicosocial busca ubicar la tamización de la depresión en el contexto de las circunstancias de la vida de cada mujer, proporcionando un enfoque holístico integrado, centrado en su salud mental. Al llevar a cabo una evaluación psicosocial más completa, el profesional puede identificar a las mujeres con vulnerabilidades (aumentadas o reducidas) que las lleven a desarrollar problemas o trastornos mentales. Adicionalmente, dada la comorbilidad común de la ansiedad con la depresión perinatal, una evaluación psicosocial integral brinda la oportunidad de progresar a una evaluación diagnóstica de posibles trastornos de ansiedad (22).

\section{Estrategias de detección y atención de los problemas o trastornos mentales durante el período perinatal}

Se encontró que varios investigadores, así como sistemas de salud de algunos países, se han dado a la tarea de diseñar, aplicar y evaluar guías o protocolos. En general, las distintas propuestas han empleado la Escala 
de Depresión Posnatal de Edimburgo (EPDS) (23), en conjunto algunas veces con instrumentos reconocidos en el ámbito clínico y de investigación para evaluar ansiedad, tales como el Test de Zung (24) o la Escala de Beck para ansiedad (25), así como cuestionarios que recogen datos sociodemográficos y psicosociales de las mujeres (lo que algunos autores denominan "evaluación psicosocial"), y cuyos resultados sirven para identificar la presencia de factores de riesgo asociados al desarrollo de depresión o ansiedad prenatal $(12,18,26-37)$.

En este sentido, se ha hecho evidente que aplicar una sola prueba de tamización, tal como la Escala de Edimburgo no sería suficiente, puesto que aquella proporciona información acerca del estado emocional de la gestante, pero estos resultados deben ser interpretados integralmente de forma que se incluya una evaluación psicosocial que permita identificar los factores de riesgo asociados con los problemas acá referidos. Al llevar a cabo una evaluación psicosocial más completa, el profesional puede identificar a aquellas mujeres con mayor vulnerabilidad. Esto puede proporcionar un enfoque preventivo útil para que las mujeres que no presentan riesgos bajo una escala como la EPDS, también puedan beneficiarse de la mayor vigilancia. Integrar la detección de posibles síntomas a la evaluación psicosocial permite vislumbrar de forma más amplia el contexto de vida de una mujer, incluidos sus apoyos, factores de estrés, la calidad de sus relaciones y su contexto cultural particular (22). En conclusión, los protocolos de atención que incluyen la evaluación psicosocial junto con la aplicación de instrumentos de tamización, especialmente para la depresión, permiten calificar el riesgo de las mujeres y así, la toma de decisiones con relación a la remisión hacia atención especializada. Esto garantizaría que estas reciban intervención oportuna y minimiza el riesgo de complicaciones futuras tanto para ellas como para sus hijos.

En el año 2016 se publicó una revisión sobre los protocolos empleados en atención primaria, entendida desde la mirada de la OMS, para la detección y tratamiento de la depresión en mujeres embarazadas y en posparto, con el fin de ayudar al Grupo de Trabajo de Servicios Preventivos de los Estados Unidos (USPSTF) a actualizar su recomendación sobre la detección de depresión en este grupo poblacional, ampliando las recomendaciones al incorporar evidencia relacionada con mujeres embarazadas y en posparto (38). El objetivo del estudio fue revisar sistemáticamente los beneficios y posibles daños de la detección y el tratamiento de la depresión para las mujeres embarazadas y en período posparto, así como la precisión de los instrumentos de detección seleccionados.
La evidencia directa e indirecta analizada por los autores sugiere que la tamización para la depresión en mujeres embarazadas y en postparto, con o sin apoyo relacionado con tratamientos adicionales (psicoterapia o farmacoterapia), reduce la prevalencia de la depresión y aumenta la remisión o la respuesta al tratamiento. La mayoría de los ensayos de detección incluidos en la revisión proporcionaron elementos de tratamiento más allá del examen, tales como capacitación y apoyo clínico, protocolos de tratamiento o asesoramiento con médicos especialmente capacitados. En mujeres embarazadas y en posparto, hubo reducciones de entre el $18 \%$ y el $59 \%$ de riesgo de desarrollar depresión en el seguimiento, en comparación con la atención habitual, lo que se tradujo en reducciones absolutas del $2.1 \%$ al $9.1 \%$ en la prevalencia de depresión, de acuerdo con la variabilidad en los puntos de corte de la Escala de Depresión de Edimburgo (38).

Frente a la revisión de la literatura previamente descrita, se hace claro que los protocolos de atención a la salud mental de las mujeres en estado de gestación incrementan la probabilidad de identificación de posibles casos clínicos que requieran un tipo de atención profesional más especializada, lo que finalmente impactaría de manera positiva en la salud de la madre, el bebé y su entorno inmediato. Ahora bien, si se identifican tanto factores de riesgo psicosociales como puntuaciones altas en las escalas de depresión y ansiedad, se hace necesaria la remisión a atención especializada, sea psicología clínica o psiquiatría, para determinar las estrategias de intervención individual indicadas. En resumen, la identificación temprana se convierte en una herramienta de acción inmediata que podrá prevenir desenlaces adversos a futuro.

\section{Componentes del protocolo de tamización de síntomas de depresión y ansiedad en el período prenatal dirigido a usuarias del servicio de control prenatal del Hospital Local del Norte (Bucaramanga)}

La primera parte del protocolo tiene por objetivo sensibilizar a los profesionales sobre la necesidad e importancia de evaluar y atender el estado de salud mental de la gestante, para lo cual se presenta información relacionada con:

- A quién va dirigido: a profesionales de la salud que acompañan a las mujeres durante el periodo de gestación.

- Propósitos: detectar o descartar de forma oportuna factores de riesgo para el desarrollo de trastornos de ansiedad o depresión en las mujeres embarazadas 
que asisten a control prenatal en el Hospital Local del Norte y tomar decisiones oportunas sobre dichos factores.

- Momento de aplicación: preferiblemente desde el segundo control prenatal o en el momento en el que el juicio del profesional de la salud lo considere necesario.

La segunda parte del protocolo guía al profesional en la tarea de detección y análisis de los factores de riesgo asociados al inicio de síntomas de depresión o ansiedad. La selección de dichos factores obedeció a la revisión de la literatura y los resultados de la investigación previamente realizada en Bucaramanga con la misma población (14). Dentro de los factores de riesgo de mayor importancia que fueron incluidos para su identificación en el protocolo se encuentran: el deseo y planeación del embarazo, haber experimentado situaciones de violencia, antecedentes psiquiátricos familiares o personales, consumo de alcohol o sustancias psicoactivas durante el embarazo, variables relacionadas con el apoyo emocional y económico y dificultades de convivencia y acontecimientos vitales estresantes (Tabla 5).

Tabla 5. Factores de riesgo incluidos en el protocolo de tamización.

\begin{tabular}{ll}
\hline Factor de riesgo & $\begin{array}{l}\text { Grado de } \\
\text { riesgo }\end{array}$ \\
\hline El embarazo fue planeado & $\mathrm{RM}$ \\
\hline El embarazo fue deseado & $\mathrm{RM}$ \\
\hline Ha sido víctima de violencia verbal durante el último año & $\mathrm{RM}$ \\
\hline Ha sido víctima de violencia física durante el último año & $\mathrm{RA}$ \\
\hline Ha sido víctima de violencia psicológica durante el último año & $\mathrm{RA}$ \\
\hline Existen antecedentes familiares de depresión y/o ansiedad & $\mathrm{RM}$ \\
\hline Ha experimentado episodios depresivos o ansiosos alguna vez en la vida & $\mathrm{RA}$ \\
\hline Tiene un diagnóstico previo de trastorno de ansiedad o depresión realizado por un profesional de la & $\mathrm{RA}$ \\
salud (Psicólogo y/o psiquiatra) & $\mathrm{RM}$ \\
\hline Ha consumido alcohol de forma regular durante el embarazo & $\mathrm{RM}$ \\
\hline Ha consumido sustancias psicoactivas durante el embarazo & $\mathrm{RM}$ \\
\hline Existen problemas de convivencia en el núcleo familiar & $\mathrm{RM}$ \\
\hline Percibe un adecuado apoyo social & $\mathrm{RM}$ \\
\hline Tiene una relación de pareja estable & $\mathrm{RA}$ \\
\hline Cuenta con apoyo emocional de su pareja & $\mathrm{RM}$ \\
\hline Cuenta con apoyo emocional de su familia & $\mathrm{RM}$ \\
\hline Cuenta con apoyo económico por parte de su pareja y/o familia & $\mathrm{RM}$ \\
\hline Actualmente existen conflictos con su pareja & $\mathrm{RM}$ \\
\hline Existe preocupación por la situación económica de la familia & $\mathrm{RA}$ \\
\hline $\begin{array}{l}\text { Presencia de acontecimientos vitales estresantes durante el último año, tales como: dificultades } \\
\text { financieras, pérdida de alguien cercano, enfermedad grave propia o de algún familiar, pérdida de } \\
\text { trabajo, entre otras. }\end{array}$ & \\
\hline & \\
\hline Ria & \\
\hline
\end{tabular}

RM: Riesgo moderado. RA: Riesgo alto

Fuente: Elaboración propia de los autores 
Con la presencia de uno o más factores de riesgo de grado alto o de tres o más de riesgo moderado, el profesional debe aplicar la EDPS. Esta ha sido probada, traducida y utilizada en más de 23 países para identificar a las mujeres que están en riesgo de depresión posparto (23), y también ha sido ampliamente validada como una herramienta efectiva de tamizaje para la depresión prenatal que puede presentarse en el primer, segundo o tercer trimestre del embarazo $(39,40)$. La EPDS es una escala de 10 ítems que requiere aproximadamente 5 minutos para completarse. Tiene una puntuación máxima de 30 , un punto de corte de 11 a 12 , una sensibilidad de $76.7 \%$ y una especificidad del $92.5 \%$ para predecir el riesgo de depresión. Esta escala no confirma un diagnóstico de depresión, pero ha mostrado su utilidad como herramienta de tamizaje que indica en sus resultados algún riesgo de un episodio depresivo mayor. Se emplea la EDPS dado que, en la revisión de la literatura correspondiente, tanto de estudios de prevalencia como de propuestas de detección, ha sido la de más amplio uso. De hecho, en la revisión consultada sobre los protocolos empleados en atención primaria para la detección y tratamiento de la depresión en mujeres embarazadas y en posparto (38), recomendaron al USPSTF el empleo específico de esta prueba.

Al mismo tiempo, se debe aplicar la Escala de Ansiedad de Zung; un instrumento que consta de veinte afirmaciones que cuantifican síntomas ansiosos, 15 somáticos y 5 cognoscitivos durante los últimos treinta días. Esta escala presenta un patrón de respuesta tipo Likert que se contesta con las opciones: "nunca", "a veces", "casi siempre" y "siempre". A cada pregunta se le da una puntuación de 1 a $4,50 \%$ en sentido positivo y $50 \%$ en sentido inverso. En consecuencia, el puntaje total puede oscilar entre 20 y 80 puntos. Para el presente estudio se consideraron los puntajes mayores a 40 como indicadores de ansiedad, teniendo en cuenta los resultados de análisis de consistencia interna (alfa de Cronbach de 0.77) en un estudio con población colombiana (41).

De acuerdo con las puntuaciones obtenidas, el profesional debe decidir la ruta de atención adecuada y determinar el nivel de apoyo requerido así:

- Si la gestante obtiene una puntuación por debajo de 13 en la escala de Edimburgo y por debajo de 40 en Zung, pero logra identificar algunos síntomas tales como la alteración en patrón de sueño e ingesta y labilidad emocional, el profesional debe emplear intervenciones de primer orden como la psicoeducación, el fortalecimiento de la red de apoyo y el fomento de un estilo de vida saludable.
- Si la gestante obtiene una puntuación igual o mayor a 13 en la escala de Edimburgo e igual o mayor a 40 en Zung, el profesional debe remitirla a valoración por psicología o psiquiatría. Lo mismo sucede si la puntuación en la pregunta 10 en la EDPS (que evalúa ideación suicida) es mayor a "0" (cero).

Igualmente, dentro de las acciones a seguir por parte del profesional se recomienda:

- Realizar psicoeducación sobre las posibles alteraciones del estado de ánimo que se pueden presentar en este período.

- Promover estilos de vida saludable, ya que el ejercicio, la buena alimentación y el sueño de calidad, entre otros hábitos saludables, pueden proteger a la mujer embarazada de desarrollar trastornos como ansiedad o depresión, e incluso minimizar algunos síntomas.

- Favorecer la activación de la red de apoyo de la gestante, puesto que la compañía de las personas significativas es una herramienta valiosa para enfrentar los retos que supone el embarazo.

- Remitir al psicólogo clínico, el cual debe profundizar el proceso de evaluación por medio de la entrevista clínica o la aplicación de pruebas específicas que permitan confirmar o descartar un diagnóstico de trastorno depresivo, ansioso $\mathrm{u}$ otros. Este profesional deberá decidir el tipo de intervención psicológica que requiere la futura madre o remitir a psiquiatría, si considera que el caso así lo amerita.

- En aquellos casos en los que la sintomatología es grave y existe riesgo para la integridad de la madre o el bebé, se debe hacer la remisión inmediata a psiquiatría.

El protocolo está acompañado de una batería de evaluación que contiene: cuestionario de datos sociodemográficos, tabla de factores de riesgo y escalas a aplicar con sus correspondientes criterios de calificación. Por último, y para facilitar el proceso de toma de decisiones, se presenta un algoritmo que guiará al profesional durante todo el proceso (Figura 1).

\section{Conclusiones}

Los trastornos del estado de ánimo y de ansiedad perinatales se encuentran entre las afecciones mentales más comunes que enfrentan las mujeres en edad reproductiva. Cuando no son tratados, pueden tener profundos efectos adversos en las mujeres y sus hijos, que van desde el aumento del riesgo de adherencia a 


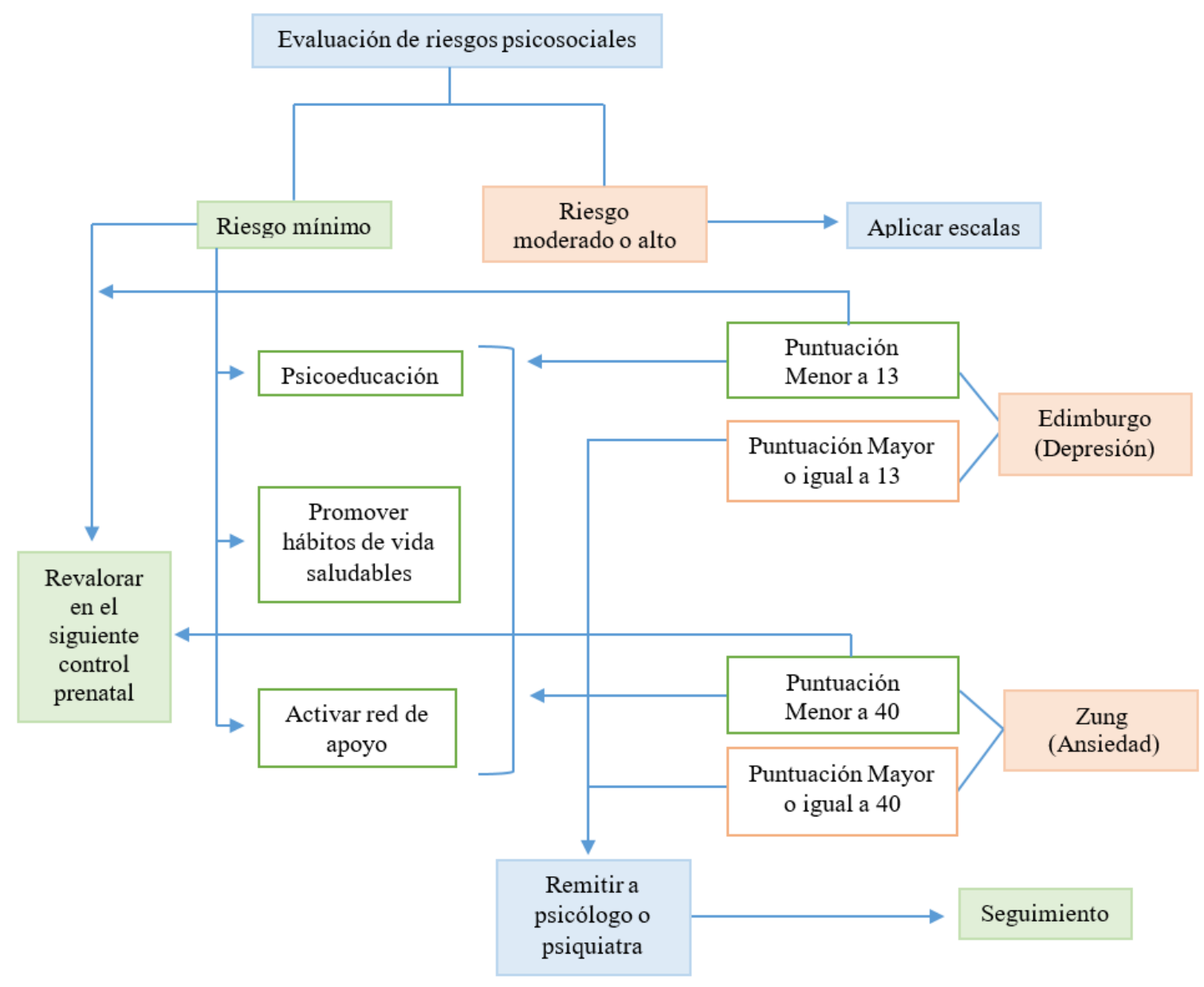

Figura 1. Árbol de decisiones

Fuente: Elaboración propia de los autores

la atención médica, la exacerbación de condiciones médicas físicas, la pérdida de recursos interpersonales $\mathrm{y}$ financieros y el consumo de tabaco y sustancias psicoactivas, hasta el suicidio o infanticidio. Los trastornos del estado de ánimo y de ansiedad perinatales están asociados con un mayor riesgo de mortalidad y morbilidad materna e infantil y se reconocen como un problema importante de seguridad del usuario. Frente a esta realidad y la importancia de un componente de atención obstétrica de calidad, se hace evidente la necesidad de incluir acciones de tamización universal para los síntomas de depresión, ansiedad y factores de riesgo para el desarrollo de trastornos mentales. Sin embargo, la tamización por sí sola no mejora los resultados perinatales, por lo que los sistemas de salud tendrían que ajustar sus protocolos de atención con el fin de garantizar una detección sistemática de dificultades de salud mental en las gestantes, para lo cual deben contar con herramientas de evaluación, así como intervenciones y procesos de seguimiento adecuados y confiables.
La valoración de la eficacia, eficiencia y efectividad de los programas de tamización y evaluación psicosocial prenatal no arroja datos concluyentes. En este sentido, quizá una de las principales dificultades de su evaluación se asocie a la debilidad de los diseños metodológicos empleados en las investigaciones, lo que en muchos casos afecta la calidad de los estudios y por ende sus resultados. Ahora bien, a pesar de no encontrar consenso sobre la eficacia, eficiencia y efectividad de las tareas de tamización y evaluación psicosocial en el periodo prenatal, en términos de morbilidad mental, es evidente que cualquier acción que se tome en el ambiente clínico y que conduzca, tanto a la detección como a la atención de dificultades en el campo de la salud mental de la futura madre, tendrá un impacto positivo sobre su salud, la de su bebé y la de su entorno.

Somos conscientes de que en la actualidad nuestro sistema de salud no posee un esquema oportuno de atención a los problemas y trastornos mentales, lo cual se 


\section{Referencias}

traduce en una posible limitación para la implementación del protocolo, ya que requiere de voluntad institucional, así como de profesionales a cargo de la atención. Para el caso específico de la población gestante, se deberían poner en marcha acciones de prevención sencillas que pueden ser incluidas en los controles prenatales, de forma que se garantice, en principio, la identificación de individuos en riesgo, para poder hacer la derivación respectiva y evitar así el impacto negativo de un estado de salud mental alterado. El protocolo aquí presentado intenta dar respuesta al vacío de identificación y atención a las dificultades de salud mental que puedan presentar las gestantes, por lo cual se convierte en una herramienta valiosa para los profesionales de la salud que atienden esta población.

Es igualmente importante destacar que llegar hasta este producto final fue resultado de un ejercicio investigativo que inició con el interés de una de las investigadoras en profundizar sobre temas de salud mental de las gestantes; población a la que prestaba sus servicios como docente asesora de estudiantes en práctica. Es así como, partiendo de la revisión de la literatura sobre salud mental en gestantes, el equipo investigador se concentra en las dos entidades con mayor prevalencia (depresión y ansiedad), identificando factores etiológicos y de mantenimiento y formas de evaluación e intervención. Tal información llevó al desarrollo de la investigación de prevalencia de ansiedad y depresión mencionada al inicio del presente artículo, cuyos resultados finalmente sirvieron de base para la construcción del protocolo presentado.

Por último, se reconoce que inicialmente se debe observar el comportamiento del protocolo en el contexto regional. Sería importante el desarrollo una investigación que evalúe la efectividad, eficiencia y eficacia del mismo. De esta manera se podrían realizar ajustes y, a mediano plazo, replicar su aplicación en el contexto nacional; una amplia validación podría llevar a sugerir a las entidades nacionales de salud la inclusión dentro de las acciones existentes de atención a las mujeres gestantes. Se espera que a mediano y largo plazo el protocolo ayude a mejorar la calidad de vida de las gestantes, sus hijos y entorno cercano, y que igualmente sirva de base al diseño e implementación de programas de prevención que minimicen el riesgo de desarrollar patologías mentales y su impacto negativo sobre la vida de las mujeres y su descendencia.

\section{Conflicto de intereses}

Los autores declaran que no tiene ningún conflicto de interés.
1. Glover V, Kammerer M. The biology and pathophysiology of peripartum psychiatric disorders. Prim Psychiatry [Internet]. 2004 [citado 23 de enero de 2018];11(3):37-41. Recuperado a partir de: https:// bit.ly/2mH8xNu

2. GloverV.Maternaldepression, anxietyand stressduring pregnancy and child outcome; what needs to be done. Best Pract Res Clin Obstet Gynaecol. 2014;28(1):2535. doi: 10.1016/j.bpobgyn.2013.08.017

3. Chung TK, Lau TK, Yip AS, Chiu HF, Lee DT. Antepartum Depressive Symptomatology Is Associated With Adverse Obstetric and Neonatal Outcomes. Psychosom Med 2001;63(5):830-834. doi: 10.1097/00006842-200109000-00017

4. Davis E, Snidman N, Wadhwa P, Glynn L, Dunkel Schetter C, Sandman, C. Prenatal Maternal Anxiety and Depression Predict Negative Behavioral Reactivity in Infancy. Infancy 2004;6(3):319-331. doi: 10.1207/s15327078in0603 1

5. Dayan J,CreveuilC,Marks MN, Conroy S, Herlicoviez M, Dreyfus M, Tordjman S. Prenatal Depression, Prenatal Anxiety, and Spontaneous Preterm Birth: A Prospective Cohort Study Among Women With Early and Regular Care. Psychosom Med 2006; 68(6):938946. doi: 10.1097/01.psy.0000244025.20549.bd

6. Grote NK, Bridge JA, Gavin AR, Melville JL, Iyengar S, Katon WJ. A Meta-analysis of Depression During Pregnancy and the Risk of Preterm Birth, Low Birth Weight, and Intrauterine Growth Restriction. Arch Gen Psychiatry 2010;67(10):1012-1024. doi: 10.1001/ archgenpsychiatry.2010.111

7. Hoffman S, Hatch M. Depressive Symptomatology During Pregnancy: Evidence for an Association With Decreased Fetal Growth in Pregnancies of Lower Social Class Women. Health Psychol 2000;19(6):535543. doi: 10.1037/0278-6133.19.6.535

8. Li D, Liu L, Odouli R. Presence of depressive symptoms during early pregnancy and the risk of preterm delivery: a prospective cohort study. Hum Reprod 2009;24(1):146-153. doi: 10.1093/humrep/ den342

9. O'Connor TG, Heron J, Golding J, Beveridge M, Glover V. Maternal antenatal anxiety and children's behavioural/emotional problems at 4 years: Report from the Avon Longitudinal Study of Parents and Children. Br J Psychiatry 2000;180(6):502-508. doi: 10.1192/bjp.180.6.502

10. Orr S, James S, Blackmore Prince S. Maternal Prenatal Depressive Symptoms and Spontaneous Preterm Births among African-American Women in Baltimore, Maryland. Am. J. Epidemiol 2000;156(9):797-802. doi: 10.1093/aje/kwf131 
11. Surkan P, Kennedy C, Hurley K, Black, M. Maternal depression and early childhood growth in developing countries: systematic review and meta-analysis. Bull World Health Organ 2011;89:608-615E. doi: 10.2471/BLT.11.088187

12. Kingston D, Austin MP, Hegadoren K, McDonald S, Lasiuk G, McDonald S, Heaman M, Biringer A, Sword W, Giallo R, Patel T, Lane-Smith M, van Zanten SV. Study protocol for a randomized, controlled, superiority trial comparing the clinical and cost- effectiveness of integrated online mental health assessment-referral-care in pregnancy to usual prenatal care on prenatal and postnatal mental health and infant health and development: the Integrated Maternal Psychosocial Assessment to Care Trial (IMPACT). Trials. 2014;6(15):72. doi: 10.1186/17456215-15-72

13. Atif N, Lovell K, Rahman A. Maternal mental health: The missing " $\mathrm{m}$ " in the global maternal and child health agenda. Semin Perinatol. 2015;39(5):345-52. doi: 10.1053/j.semperi.2015.06.007

14. Osma Zambrano SE, Lozano Osma MD, Mojica Perilla M, Redondo Rodriguez S. Prevalencia de depresión y ansiedad y variables asociadas en gestantes de Bucaramanga y Floridablanca (Santander, Colombia). MedUNAB. 2019;22(2):171185. doi:10.29375/0123-7047.3586

15. Ministerio de la Protección Social, Colciencias. Centro de Estudios e Investigación en Salud de la Fundación Santa Fé de Bogotá, Escuela de Salud Pública de la Universidad de Harvard. Bogotá: Guía Metodológica para el desarrollo de Guías de Atención Integral en el Sistema General de Seguridad Social en Salud Colombiano; 2010.

16. Gouda R, Hasman A, Strijbis AM, Peek N. A parallel guideline development and formalization strategy to improve the quality of clinical practice guidelines. International Journal of Medical Informatics. 2009;78:513-520. doi:10.1016/j. ijmedinf.2009.02.010

17. Swinglehurst D. Evidence-based guidelines: The theory and the practice. Evidence-Based Healthcare \& Public Health. 2005;9:308-314. doi:10.1016/j. ehbc.2005.05.012

18. Austin MP, Highet N and the Guidelines Expert Advisory Committee. Clinical practice guidelines for depression and related disorders - anxiety, bipolar disorder and puerperal psychosis - in the perinatal period. A guideline for primary care health professionals. Melbourne: beyondblue: the national depression initiative; 2011.

19. Biaggi A, Conroy S, Pawlby S, Pariante CM. Identifying the women at risk of antenatal anxiety and depression: A systematic review. Journal of
Affective Disorders. 2016;19: 62-77. doi:10.1016/j. jad.2015.11.014

20. Coates AO, Schaefer CA, Alexander JL: Detection of postpartum depression and anxiety in a large health plan. J Behav Health Serv Res. 2004;31:117-133. doi: 10.1007/BF02287376

21. Spitzer RL, Williams JB, Kroenke K, Hornyak R, McMurray J: Validity and utility of the PRIME-MD patient health questionnaire in assessment of 3000 obstetric-gynecologic patients: the PRIME-MD Patient Health Questionnaire Obstetrics-Gynecology Study. Am J Obstet Gynecol. 2000;183:759-769 doi: 10.1067/mob.2000.106580

22. Milgrom, J. \& Gemmill, A.W. (2014). Screening for perinatal depression. Best Practice \& Research Clinical Obstetrics and Gynaecology. 28:13-23. doi: 10.1016/j.bpobgyn.2013.08.014

23. Cox JL, Holden JM, Sagovsky R. Detection of postnatal depression: development of the 10-item Edinburgh Postnatal Depression Scale. Br J Psychiatry. 1987;150:782-876. doi: 10.1192/bjp.150.6.782

24. Zung WW. A rating instrument for anxiety disorders. Psychosomatics. 1971;12(6):371-9. doi: 10.1016/ S0033-3182(71)71479-0

25. Beck AT, Brown G, Epstein N y Steer RA. An Inventory for Measuring Clinical Anxiety: Psychometric Properties. Journal of Consulting and Clinical Psychology 1.988; 56:893-7.

26. Austin MP, Middleton P, Reilly NM, Highet NJ. Detection and management of mood disorders in the maternity setting: the Australian Clinical Practice Guidelines. Women Birth 2013;26(1):2-9. doi: 10.1016/j.wombi.2011.12.001

27. Carroll JC, Reid AJ, Biringer A, Midmer D, Glazier RH, Wilson L, Permaul JA, Pugh P, Chalmers B, Seddon F, Stewart DE. Effectiveness of the Antenatal Psychosocial Health Assessment (ALPHA) form in detecting psychosocial concerns: a randomized controlled trial. CMAJ. 2005;173(3):253-259. doi: 10.1503/cmaj.1040610

28. Honikman S, van Heyningen T, Field S, Baron E, Tomlinson M. Stepped care for maternal mental health: a case study of the perinatal mental health project in South Africa. PLoS Med. 2012; 9(5): e1001222. doi: 10.1371/journal.pmed.1001222

29. National Institute for Health and Clinical Excellence (NICE). Antenatal and postnatal mental health: The NICE guidelines on clinical management and service guidance CG45. National Collaborating Centre for Mental Health. The British Psychological Society \& The Royal College of Psychiatrists; 2007.

30. National Institute for Health and Care Excellence. Antenatal and postnatal mental health: clinical management and service guidance. NICE clinical 
guideline 192. London: The British Psychological Society \& The Royal College of Psychiatrists; 2014.

31. Thoppil J, Riutcel TL, Nalesnik SW. Early intervention for perinatal depression. Am J Obstet Gynecol. 2005;192(5):1446-8. doi: 10.1016/j.ajog.2004.12.073

32. Austin M-P, Hadzi-Pavlovic D, Leader L, Saint K, Parker G. Antenatal screening for the prediction of postnatal depression: validation of a psychosocial Pregnancy Risk Questionnaire. Acta Psychiatrica Scandinavica 2005;112(4):310-7. doi: 10.1111/j.16000447.2005.00594.x

33. Austin MP, Colton J, Priest S, Reilly N, HadziPavlovic D. The antenatal risk questionnaire (ANRQ): acceptability and use for psychosocial risk assessment in the maternity setting. Women Birth. 2013; 26(1):1725. doi: 10.1016/j.wombi.2011.06.002.

34. Gordon TE, Cardone IA, Kim JJ, Gordon SM, Silver RK. Universal perinatal depression screening in an Academic Medical Center. Obstet Gynecol. 2006;107:342-7. doi: 10.1097/01. AOG.0000194080.18261.92

35. SIGN. Management of perinatal mood disorders (SIGN Publication no. 127). [Internet] Edinburgh: Scottish Intercollegiate Guidelines Network; 2012 [citado el 23 de enero de 2018]. Recuperado a partir de: http:/www.sign.ac.uk/assets/sign127.pdf

36. Segre LS, O'Hara MW, Brock RL, Taylor D. Depression screening of perinatal women by the Des Moines Healthy Start Project: program description and evaluation. Psychiatr Serv. 2012;63(3):250-5. doi: 10.1176/appi.ps.201100247.

37. Ministerio De Salud. Protocolo de Detección de la Depresión durante el Embarazo y Posparto, y Apoyo al Tratamiento; 2014 [citado 23 de enero de 2018] Santiago: Minsal. Recuperado a partir de: https://bit. ly/2laVjbn

38. O'Connor E, Rossom RC, Henninger M, Groom HC, Burda BU. Primary Care Screening for and Treatment of Depression in Pregnant and Postpartum Women. Evidence Report and Systematic Review for the US Preventive Services Task Force. JAMA 2016; 315(4):388-406. doi:10.1001/jama.2015.18948

39. Gaynes, BN., Gavin, N., Meltzer-Brody, S., Lohr, KN., Swinson, T., Gartlehner, G., Brody, S. \& Miller, WC. Perinatal Depression: Prevalence, Screening Accuracy, and Screening Outcomes. Evidence Report/ Technology Assessment. [Internet] 2005 [citado 23 de enero de 2018];(119):1-8. Recuperado a partir de: https://bit.ly/2laY9x3

40. Gibson, J., McKenzie-McHarg, K., Shakespeare, J., Price, J. \& Gray, R. A systematic review of studies validating the Edinburgh Postnatal Depression Scale in antepartum and postpartum women. Acta Psychiatr Scand 2009;119(5):350-64. doi: 10.1111/j.16000447.2009.01363.x.

41. De la Ossa, S.; Martinez, Y.; Herazo, E. \& Campo, A. Estudio de la consistencia interna y estructura factorial de tres versiones de la escala de Zung para ansiedad. Colomb. Med. [Internet]. 2009 [citado 23 de enero de 2019];40(1):71-77. Recuperado a partir de: https://bit. ly/2la81ab 